\title{
Love you to. Un exemple de rencontre entre musique indienne et musique pop dans la production des Beatles
}

\section{Laura Leante}

Traducteur : Georges Goormaghtigh

\section{OpenEdition}

\section{Journals}

Édition électronique

URL : http://journals.openedition.org/ethnomusicologie/696

ISSN : 2235-7688

Éditeur

ADEM - Ateliers d'ethnomusicologie

Édition imprimée

Date de publication : 1 janvier 2001

Pagination : 103-118

ISBN : 2-8257-0723-6

ISSN : 1662-372X

Référence électronique

Laura Leante, «Love you to. Un exemple de rencontre entre musique indienne et musique pop dans la production des Beatles", Cahiers d'ethnomusicologie [En ligne], 13 | 2001, mis en ligne le 09 janvier 2012, consulté le 19 avril 2019. URL : http://journals.openedition.org/ethnomusicologie/696

Ce document a été généré automatiquement le 19 avril 2019

Tous droits réservés 


\title{
Love you to. Un exemple de rencontre entre musique indienne et musique pop dans la production des Beatles
}

\author{
Laura Leante \\ Traduction : Georges Goormaghtigh
}

1 Le métissage entre musique pop et musiques ethniques est un aspect important de la réalité musicale d'aujourd'hui; désormais omniprésent, il s'est imposé en tant que phénomène ayant profondément marqué la production discographique de ces trente-cinq dernières années.

2 La présence toujours plus massive de musiques «autres» dans le marché du pop/rock a contribué à la diffusion, vers la fin des années quatre-vingt, du terme world music, inventé pour désigner tous les genres étrangers à la tradition pop anglo-américaine ${ }^{1}$. En même temps, la prolifération des contaminations a été facilitée par les nouvelles technologies d'enregistrement et d'élaboration du son et s'est développée parallèlement à des genres comme le New Age ou l'Ambient, dont l'une des composantes essentielles est la recherche de sonorités souvent exotiques par le biais d'une utilisation importante de sons naturels, de synthétiseurs ou de la technique de l'échantillonnage.

3 Le dynamisme des transformations et des contaminations des productions pop les plus récentes a amené l'ethnomusicologie à s'intéresser aussi à ce secteur, alors qu'elle avait d'abord fait preuve d'une certaine méfiance à l'égard de la popular music, lui préférant l'étude des cultures traditionnelles².

4 Une des difficultés propres à l'approche de ce type particulier de métissage vient de ce qu'il est indispensable de connaitre les deux mondes qui s'y rencontrent pour pouvoir enquêter sur les modalités culturelles et musicales à travers lesquelles la musique pop occidentale a absorbé des éléments d'une musique «autre» ou, vice versa, comment ce même Occident a pu influencer d'autres réalités musicales. La recherche se situe par conséquent dans une zone frontière, non seulement entre les deux cultures musicales, 
mais encore entre deux disciplines, la musique pop et l'ethnomusicologie. Il est essentiel de comprendre comment les éléments «ethniques» ont été reçus par le musicien occidental et de là, intégrés dans la musique pop: le résultat de ce passage est souvent plein d'ambiguïté entre ce qui est sciemment emprunté à la musique «autre» et ce qui est absorbé et adapté à cause d'une apparente proximité avec des figures de style ou des structures occidentales.

Dans ce cadre multiforme et complexe, la rencontre entre la musique pop et la musique indienne occupe une place à part car elle a donné lieu à une production qui s'est très largement répandue, créant une série de modes et de sous-genres, récupérant ces dernières années des formes et des styles du sous-continent indien tels que l'Asian underground ou le Bhangra.

6 L'Inde pénètre dans les productions discographiques soit par fascination pour une sonorité «autre», soit comme composante plus marquée et consciente de la composition, comme c'est le cas, par exemple, de la collaboration de Zakir Hussein à de nombreuses productions rock et fusion ou encore du groupe anglo-indien des Cornershop et du tabliste et producteur Talvin Singh, qui est considéré par la critique pop britannique contemporaine comme un des défenseurs les plus représentatifs de la fusion de ces dernières années pouvant se prévaloir de nombreuses collaborations avec des stars et des musiciens occidentaux.

7 Aujourd'hui la présence d'instruments indiens ou de tournures propres à la musique indienne dans la musique pop est un fait accompli. Mais les premières tentatives de crossover remontent aux années soixante, quand certains représentants de la tradition musicale classique hindoustanie (Inde du Nord) apportèrent leur contribution essentielle au métissage. Le premier d'entre eux est le sitariste Ravi Shankar, figure clé de la médiation entre la musique indienne et le monde occidental, un des protagonistes, dans la seconde moitié de cette décennie, de la «great sitar explosion» (comme il l'a lui-même définie ${ }^{3}$ ). Il s'agit du premier cas important de contamination par des musiques «autres» dans le domaine de la musique pop, qui fit connaître au public occidental des sonorités et des musiciens appartenant à des cultures musicales extra- européennes. 


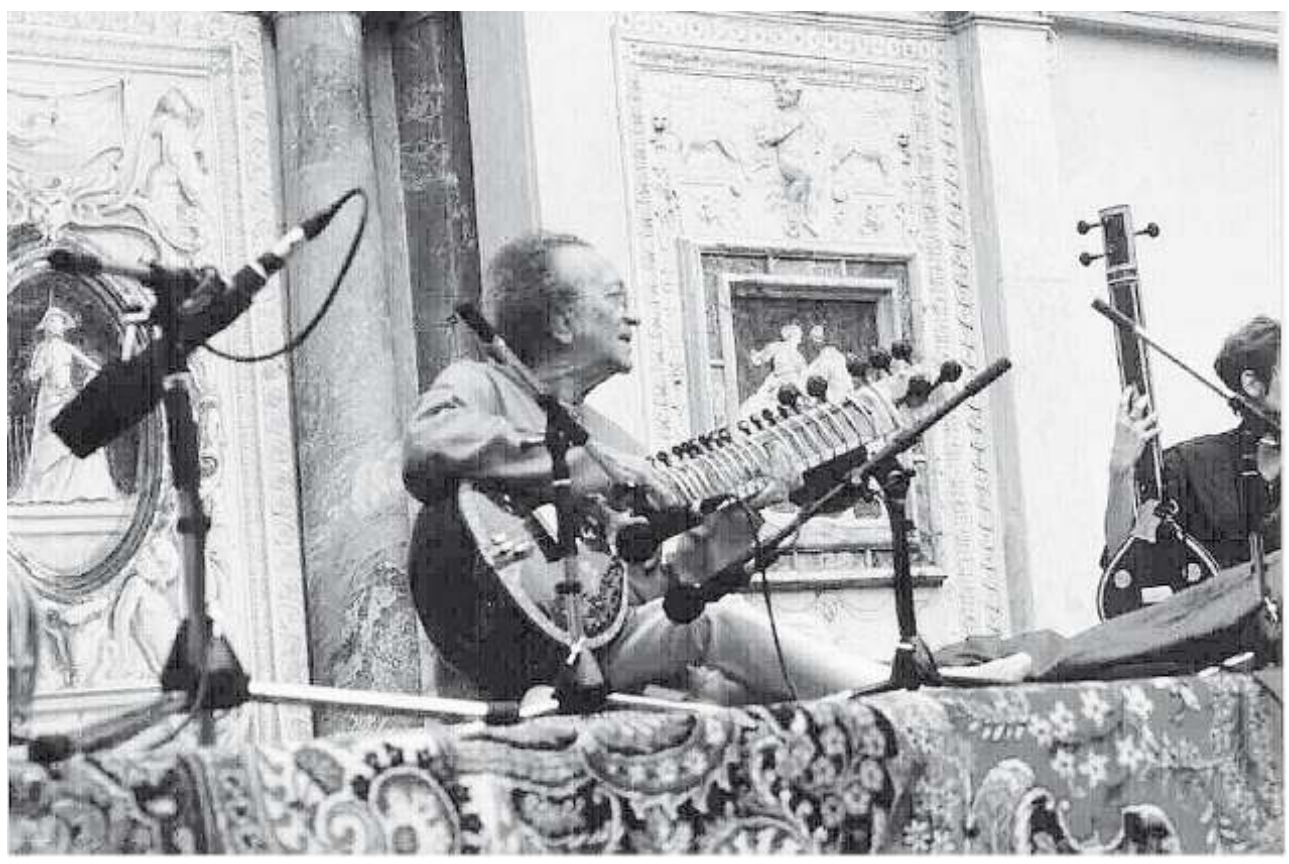

Photo: Laura Leante.

8 Le contexte est celui de la mode lancée par la contre-culture anglo-américaine de l'époque des poètes beat à Timothy Leary, qui tendait à faire croire que "psychédélique» et «Orient» étaient les deux termes d'une même équation. Ainsi, à l'expérimentation fervente tant sonore que structurelle qui caractérisait la production rock de ces années, se joignirent l'attrait pour la dimension hallucinogène et les sonorités exotiques censées lui correspondre.

Parmi les nombreuses stars de la musique pop qui s'approchèrent de la musique indienne - et en particulier du sitar - les Beatles se distinguent, surtout grâce à George Harrison, lui-même élève de Ravi Shankar, et à son intérêt sincère et durable pour la culture et la tradition musicale de l'Inde du Nord.

Le rôle joué par les Beatles dans le métissage entre la musique indienne et la musique pop occidentale a été déterminant car il était pionnier, innovateur et exemplaire pour les tendances qui suivirent. En fait, les Beatles ont été parmi les principaux agents de l'« explosion», précédant les autres musiciens pop en publiant une chanson - Norwegian Wood ${ }^{4}$ - dans laquelle était joué un instrument hindoustani. Plus que quiconque ils ont promu et diffusé ce phénomène grâce à l'énorme succès remporté par leur production tant dans les milieux musicaux créatifs qu'auprès du grand public. En outre, contrairement à leurs contemporains, ils ne se sont pas limités à l'utilisation d'instruments indiens pour obtenir un effet acoustique particulier évoquant des dimensions exotiques et hallucinogènes: ils ont mené une expérimentation plus articulée, qui, par-delà la seule recherche de sonorités spécifiques, a conditionné l'aspect structurel, mélodique et rythmique de leurs créations, en travaillant avec des échelles modales, des cycles rythmiques et des formes musicales hindoustanis.

La production du groupe entre 1965 et 1966 prouve à quel point, à l'époque déjà, les Beatles étaient entrés dans la voie de l'expérimentation, tant sur le plan structurel, avec 
la réalisation de morceaux s'écartant de la forme chanson, que sur celui de la sonorité en utilisant des instruments n'appartenant pas à l'idiome du rock et en s'appuyant sur les technologies mises à disposition dans le studio d'enregistrement. Ce fut justement la curiosité pour un sound nouveau qui les amena à recourir au sitar et au tanpura dans Norwegian Wood et Tomorrow Never Knows; dans ces deux pièces, l'utilisation d'éléments sonores «autres» est assez semblable à ce qui s'est fait dans le reste de la production pop occidentale et l'instrument indien semble utilisé uniquement pour obtenir un effet de timbre particulier, quasi-exotique pour la première et psychédélique pour la seconde.

Fig. 2: Les Beatles en Inde

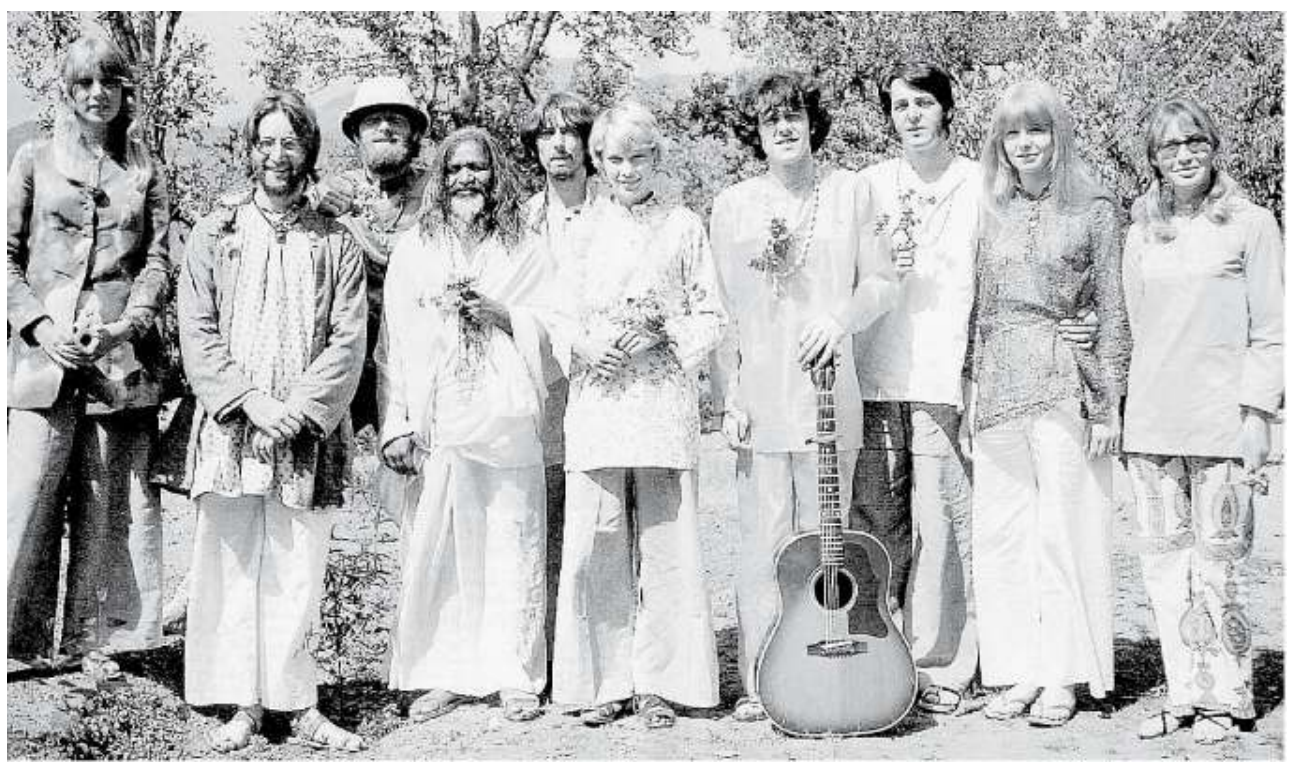

Extraite du numéro d'août 1998 du fanzine The Beatles Book monthly. Ealing, London: Beat Publications-Parker Mead Ltd.

12 C'est avec Love You To, une composition de George Harrison enregistrée en avril 1966 et destinée à figurer dans l'album Revolver que, pour la première fois, les Beatles présentent une volonté évidente d'utiliser, en plus des instruments et des sonorités hindoustanis, les formes, les échelles et les rythmes de cette musique.

Cette démarche délibérée confère tout son poids à ce morceau et va de pair avec le fait que Love You To contient déjà les éléments (structurels, modaux et rythmiques) qui allaient apparaître de diverses façons dans les autres chansons «indiennes» de Harrison ( Within You Without You et The inner light $t^{5}$ ). Le morceau est donc un bon exemple des pièces d'influence hindoustanie réalisées par les Beatles. Il est en outre intéressant de noter, comme le prouve l'analyse, combien changeante peut être la frontière entre l'acquisition intentionnelle et celle inconsciente, d'éléments «autres».

Aucun texte sur les Beatles ne présente une analyse approfondie de leur rapport avec la musique indienne ${ }^{6}$ et même les livres de Mellers (1973) et de O'Grady (1983) fondamentaux pour une étude musicologique de leur production - ne traitent pas de la question de façon précise. Les écrits de David Reck (1985) et Gerry Farrell (1990; 1997) méritent cependant d'être mentionnés comme faisant exception. L'un et l'autre se distinguent en effet car ils ont traité le thème du crossover entre la musique pop et la musique hindoustanie et analysé Love You To en adoptant une perspective ethnomusicologique. Toutefois, le premier prête à Harrison des intentions qui, dans la 
composition de la chanson, vont bien au-delà de ce que révèle une analyse précise ${ }^{7}$. Farrell, dans sa vaste étude sur la musique indienne et l'Occident, consacre deux pages à Love You To en tant que chanson emblématique du rapport entre la musique pop et la culture hindoustanie. Son analyse est centrée sur l'aspect du timbre et de la forme et cherche à déterminer, sur le plan technique et musical, quels ont été les éléments qui ont permis le métissage. Cette étude de Farrell a servi de point de départ à mon travail.

Mon analyse de Love You To fait partie d'une étude ethnomusicologique plus ample qui couvre l'ensemble de la production hindoustanie des Beatles ${ }^{8}$ et aborde différents aspects: la mélodie, le rythme, la forme et même la genèse de la chanson dans le studio d'enregistrement.

Partant d'une nouvelle transcription intégrale du morceau (jusqu'alors elle était effectuée seulement de manière approximative ou pour le moins partiellement inexacte) et d'une analyse musicale détaillée, mon but a été de comprendre comment les éléments indiens se placent dans la chanson, en m'interrogeant sur le degré de conscience que les Beatles pouvaient avoir du travail de mutation qu'ils étaient en train d'effectuer, en examinant avec soin la genèse du morceau.

17 Jusqu'à ce jour aucun chercheur n'a étudié de façon rigoureuse la question de savoir combien les Beatles étaient conscients de l'appropriation qu'ils effectuaient, c'est justement cette question, fondamentale pour moi, qui m'a menée à reconsidérer les sources, y compris Farrell.

Il est possible de reconstituer le processus d'enregistrement de la chanson avec une certaine exactitude grâce au livre de Mark Lewisohn (1988) qui donne une description précise de toutes les séances d'enregistrement des Beatles, fournissant par la même occasion de précieuses indications sur quand et comment les instruments hindoustanis furent utilisés dans Love You To. 
Fig.3: Harrison et Shankar

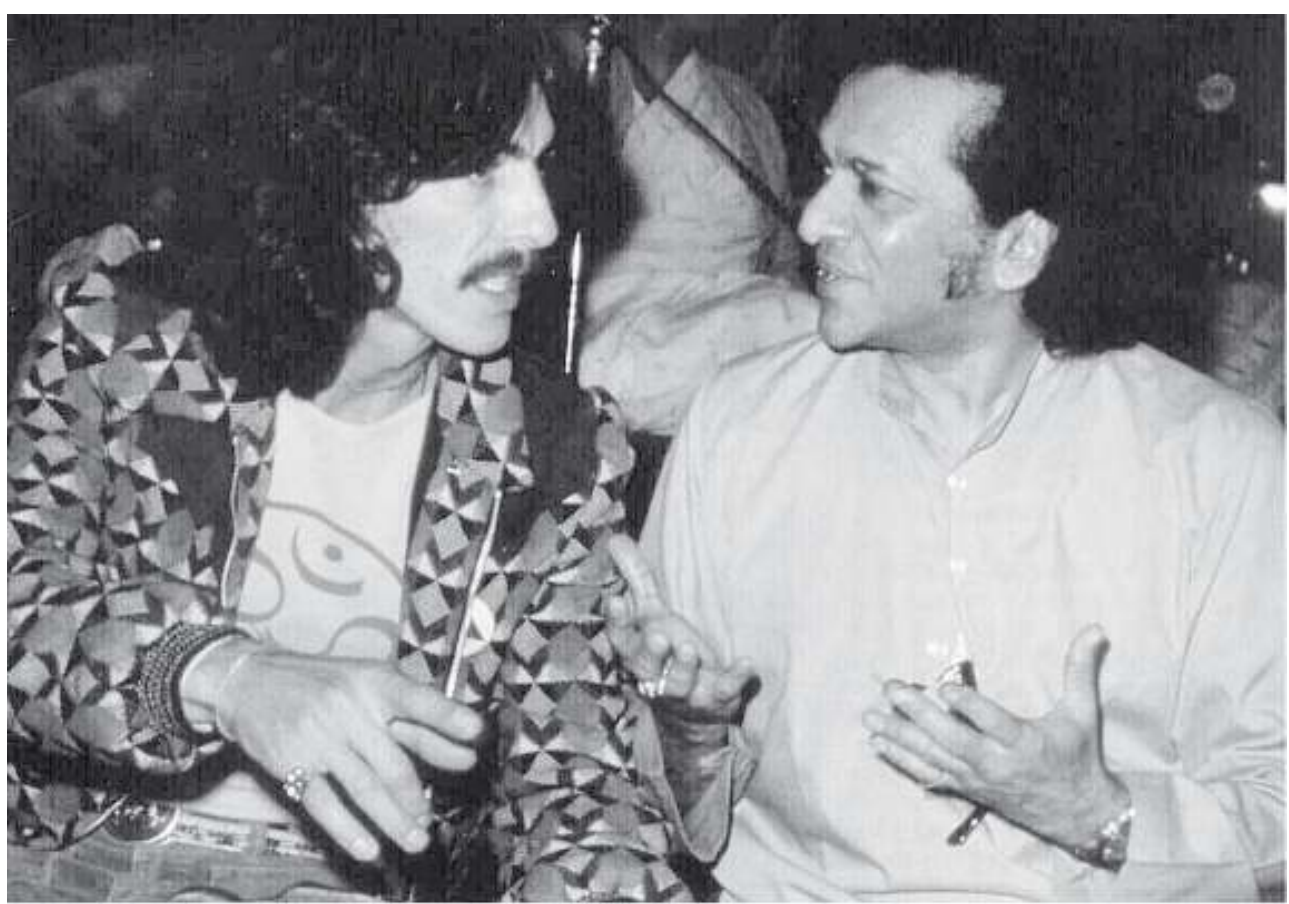

Extraite du numéro d'août 1998 du fanzine The Beatles Book monthly. Ealing, London: Beat Publications-Parker Mead Ltd.

Les enregistrements du morceau commencèrent le 11 avril 1966 et s'achevèrent, si l'on ne tient pas compte des remixages, en deux séances seulement. La première bande comportait la voix de Harrison, accompagnée par une guitare acoustique et par des chœurs de McCartney. Le sitar apparaît dans la bande 3 et, en surimpression, dans la sixième avec les tabla ${ }^{10}$. Le sitar, contrairement à ce qu'affirment certains (O'Grady 1983: 100) est joué par Harrison lui-même. Farrell fait remarquer l'accordage imprécis de l'instrument ainsi qu'une utilisation des harmoniques non conforme à la pratique indienne:

«... il y a l'utilisation d'harmoniques dans le passage introductif lent. Les harmoniques ne sont jamais utilisés comme moyen musical au sitar (ils servent à l'accordage $)^{11} \gg$ (Farrell 1988: 194).

Pour les tabla, les Beatles firent appel à un musicien indien, Anil Bhagwat. Harrison lui indiqua lui-même comment placer son exécution et participa à l'accordage du tabla, lui suggérant d'exécuter comme tala (cycle rythmique) le tintal et de se conformer au style typique de son guru, Ravi Shankar ${ }^{12}$. Bien que Bhagwat ait en principe eu le droit d'improviser comme le voulait la pratique indienne, le produit des enregistrements montre qu'en réalité il est resté très proche du theka, c'est-à-dire de l'ossature rythmique accentuée, ainsi que des frappes de base du tala utilisé ${ }^{13}$, obtenant une structure très simple comparée à ce que peut donner une exécution en contexte hindoustani et par conséquent plus facilement accessible aux oreilles occidentales.

De telles observations confirment que Harrison connaissait la théorie et la technique des tabla, et qu'il était bien conscient du résultat que le musicien devait obtenir au point de lui donner des indications non seulement de type rythmique en indiquant le tintal comme tala à utiliser, mais encore de type stylistique. 
$22 \mathrm{Au}$ cours de la seconde journée de travail, le 13 avril, on réalisa une septième bande, obtenue par la réduction de la sixième qui comportait les enregistrements précédents afin de pouvoir effectuer des surimpressions: George rajouta une autre ligne vocale et Ringo Star le tambourin, alors qu'une nouvelle contribution vocale de McCartney sur le vers «They'll fill you in with all the sins you see» fut finalement écartée au cours du mixage ${ }^{14}$.

A ce stade, il ne restait plus à Love You To qu'à subir un travail de remixage, ce qui fut fait le 21 juin suivant, pour être achevé et intégré dans l'album. Le texte de Love You To ne présente pas de caractéristiques particulières à la culture indienne, mais se veut une réflexion sur la fugacité du temps et de la vie et sur la corruption spirituelle du monde, le tout accompagné d'une allusion à l'expérience sentimentale, d'une invitation à l'amour intense comme refuge de pureté et abri dans la brièveté de l'existence.

Sur le plan musical et formel, la chanson propose certains éléments typiques de l'exécution d'un raga hindoustani ${ }^{15}$.

Comme on le sait, la forme d'un raga se compose d'un prélude en temps libre (alap), qui expose le matériau mélodique de la composition, suivi d'autres sections (gat et jhala), qui se caractérisent par l'introduction des percussions et par l'accélération constante du mètre, jusqu'à ce que l'élément rythmique devienne prépondérant.

Dans Love You To il est justement possible de reconnaître en germe les composantes essentielles de ce modèle structurel.

27 La première partie, à caractère introductif, se présente en effet comme un alap, dans lequel on peut identifier, avec l'accélération de la pulsation, les trois parties qui traditionnellement le composent: le vilambit (tempo lent), le madhya (tempo moyen) et le drut (tempo rapide).

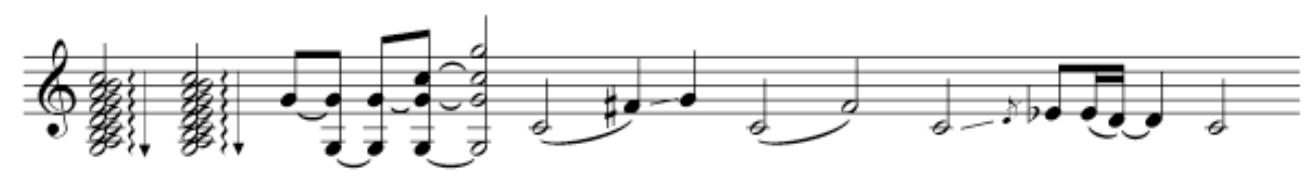

Dans cette section, le sitar expose l'échelle sur laquelle est construite la mélodie qui, à part le quatrième degré haussé, présent seulement au début de l'alap, correspond au kafi tata ${ }^{16}$ hindoustani, qu'on peut ramener au mode dorien par analogie, dans la succession des intervalles, avec la tierce et la septième mineures ${ }^{17}$ :

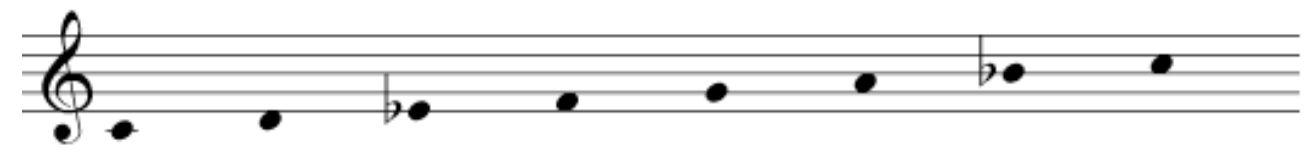

29 A l'alap succède une section gat, avec une accélération progressive et constante du mètre, grâce à l'attaque des tabla, précédée d'une phrase au sitar qui sera réexposée au cours du morceau avec une fonction similaire à celle du riff de guitare dans la pop. 


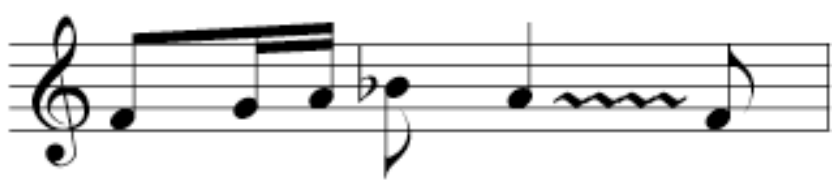

Le cycle rythmique (tala) utilisé dans la chanson est le tintal, composé de seize pulsations et confié aux tabla. Il est joué à la mesure d'un $4 / 4$ occidental, dans lequel le cycle complet est subdivisé en quatre sous-groupes de quatre pulsations chacun, où l'on ne rencontre que peu de frappes irrégulières.

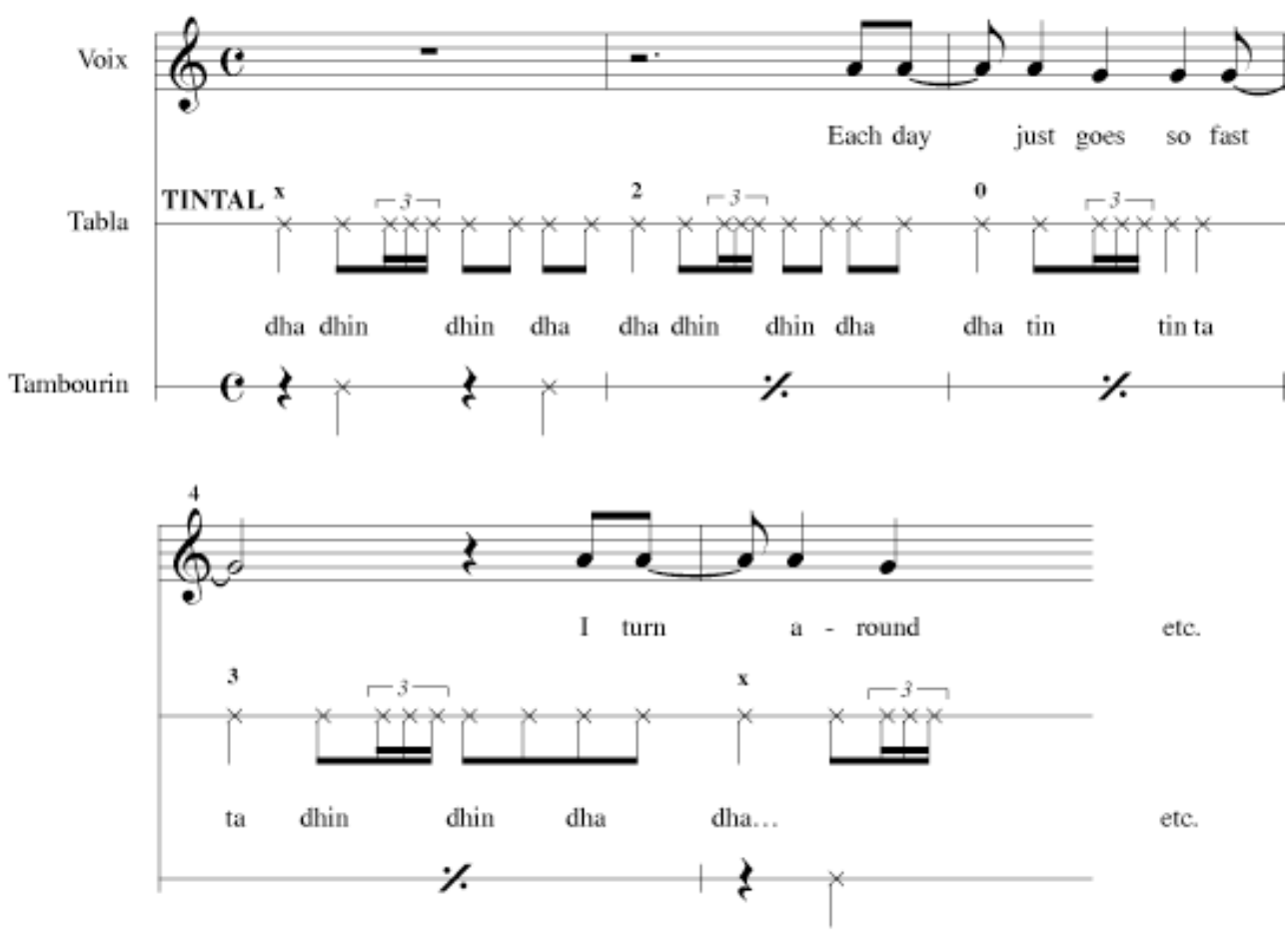

Bien que les tabla soient joués par un musicien hindoustani, et donc de manière techniquement conforme à la tradition indienne, le tala est plusieurs fois adapté aux exigences de la chanson occidentale; en effet jamais tout au long du morceau il ne termine sur le sam, première pulsation du cycle et accent principal. De la même manière, les moments de silence des tabla ne correspondent pas à un nombre déterminé de pulsations (par exemple celui d'un cycle complet), mais sont de durée variable. Ces observations sont autant de preuves de la présence d'interventions et de directives données au tabliste par Harrison.

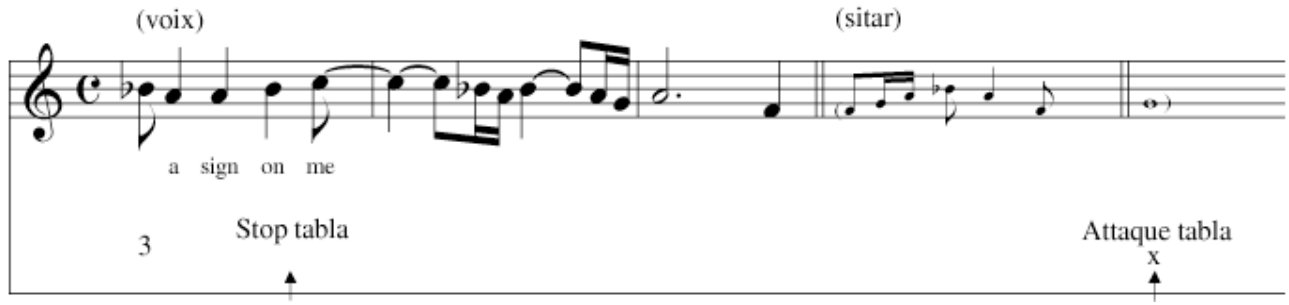

Conformément à la pratique hindoustanie, Love You To est clairement modal: sur le bourdon du tanpura, la voix et le sitar tissent la mélodie. 
в)

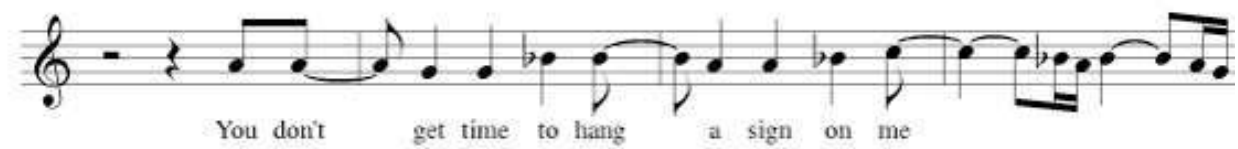

$\gamma)$

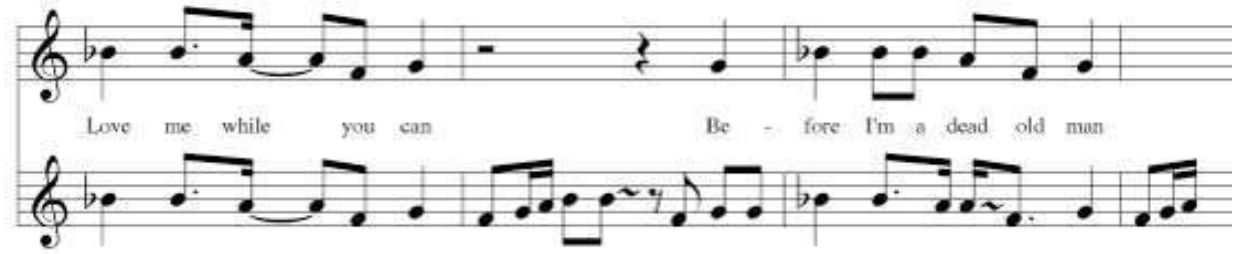

La ligne du chant comme celle du sitar s'articule sur le modèle du kafi tata et se développe fondamentalement sur trois «noyaux mélodiques».

Le premier (« $»)$ est construit, avec des notes répétées, sur un seul intervalle de seconde et est repris deux fois, créant ainsi une sorte de «dialogue» avec les interventions du sitar. Il est intéressant à ce propos de noter combien la structure qui prévoit une réponse entre deux instruments est typique de la tradition hindoustanie, mais en même temps familière à la musique pop, qui l'a empruntée au blues.

Le second noyau mélodique de Love You To («ß») se base, comme le premier, sur un matériau syncopé et se développe dans un intervalle de tierce présentant un mouvement descendant à caractère mélismatique se référant, avec le ton nasal de la voix, à la pratique chantée indienne.

Dans le troisième ("ү») la voix et le sitar réexposent (dans un intervalle de quarte) la forme responsoriale déjà présente dans le premier.

Dans la chanson figurent également un solo de sitar et une coda instrumentale. Le premier présente une fonction analogue au solo de guitare pop/rock; la seconde, caractérisée par un accelerando ultérieur, semble correspondre à la section jhala d'un raga et se termine sur un fondu résultant manifestement, quant à lui, d'une intervention en phase de mixage.

Ces deux interventions de sitar semblent vouloir explorer (sur une base souvent syncopée) les développements mélodiques de la gamme, dans un mouvement alternativement ascendant et descendant. Cet élément, ainsi que la très manifeste accélération du tempo dans la partie finale du morceau, est une autre référence à la représentation d'un raga hindoustani.

$\alpha)$

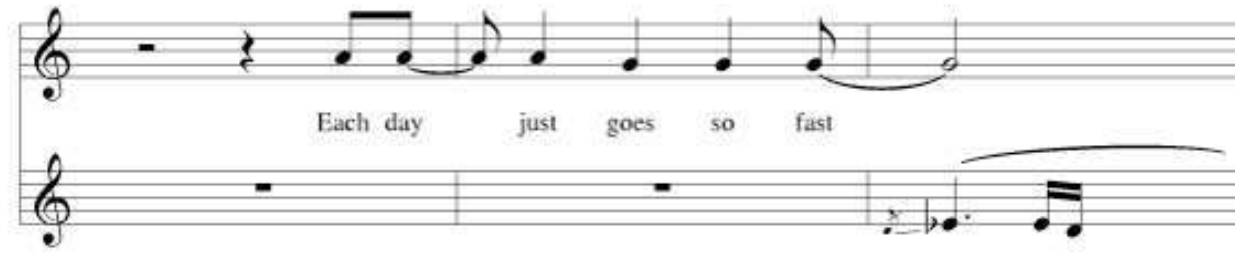

sign on me

Il est intéressant de noter que, si d'un côté le sitar, comme nous venons de le voir, tend parfois à assumer des fonctions semblables à celles typiques de la guitare dans l'idiome de la musique pop occidentale, ces brèves interventions de la guitare électrique dans Love You To renforcent, quant à elles, le substrat sonore produit par le bourdon du tanpura. 
C'est justement sur l'ambiguïté de cette présence de facteurs opposés, sur cet échange continu entre l'Est et l'Ouest qu'a lieu, chez les Beatles, le crossover entre la pop occidentale et la musique indienne. Schématiquement, la structure formelle du morceau est la suivante:

«[...] Il y a de nombreux aspects dans cette chanson qui sont bien dans la tradition du malentendu occidental au sujet de la musique indienne, même si l'arrangement était sans précédent pour une chanson pop [...] Le plus grand malentendu se trouve dans la production vocale, qui semble refléter l'idée que pour sonner indien, il faut chanter plat avec une voix nasale, plutôt que de façon coulante et expressive. Pour ce qui est de la structure, il est clair que la chanson de Harrison propose une esquisse fort simple des vastes compositions de la musique indienne qui, une fois rendues sans les détails, perdent tout leur sens ${ }^{18} »$ (Farrell 1997: 184).

Selon Farrell, l'intention de Harrison aurait été de porter la chanson pop vers de nouveaux horizons mais le résultat fut peu concluant dans la mesure où la musique indienne se trouve ramenée à une série de "formules» limitées qui ne reflètent que superficiellement ses structures et ses tournures. Ceci parce que Harrison, quoique sincèrement intéressé à la culture musicale hindoustanie, n'aurait pas encore été, à l'époque, en pleine possession de la technique et des connaissances nécessaires pour pouvoir pénétrer à fond les possibilités de développement et d'articulation interne d'un raga $^{19}$.

Des affirmations si catégoriques doivent probablement être quelque peu nuancées. Ce qu'affirme Farrell est certainement compréhensible du point de vue d'un chercheur orientaliste. Cependant, on finit peut-être par attribuer à Harrison une volonté qui va audelà de ses intentions réelles. Terence O'Grady remarque combien la position de l'auteur à l'égard de ses propres compositions d'influence indienne est dénuée de toute prétention de reproduire «toute l'essence de la tradition classique de l'Inde ${ }^{20} »$ (O'Grady 1983: 101). D'autre part, c'est le même Harrison qui déclare:

«Les vrais chants classiques indiens sont très différents du genre de chansons pop influencées par l'Inde que j'ai eu l'occasion d'entendre par ici. Ce ne sont que des chansons pop, avec un peu de fond indien.

Je ne suis pas sûr pour celles que j'ai écrites. Si je les considère du point de vue d'une autre personne, en tant que chansons pop je les aime bien» (Harrison, cit. in Davies 1985: 409)..$^{21}$

Le musicien montre donc qu'il faisait clairement la différence entre la «vraie» musique classique indienne et ses propres chansons, qui restent toujours des «pop songs». En d'autres termes, l'expérimentation est bien faite avec des moyens et des instruments indiens, mais elle reste toujours dans le cadre de la musique pop occidentale et n'a pas la prétention de condenser l'essence de la musique de l'Inde du Nord en seulement trois minutes.

D'autre part, on ne saurait blâmer Farrell pour son ton péremptoire si l'on se rappelle que son intention était de réfuter, à son tour, les affirmations de ceux qui, comme Reck (1985: 
102), considèrent Love You To avec enthousiasme et proclament à tort des résultats basés sur des buts présumés, mais - comme on l'a vu - dénués de fondements, de synthétiser la musique hindoustanie.

Fig. 4: Pochette de l'album Revolver (Parlophone-EMI Records Ltd, 1966).

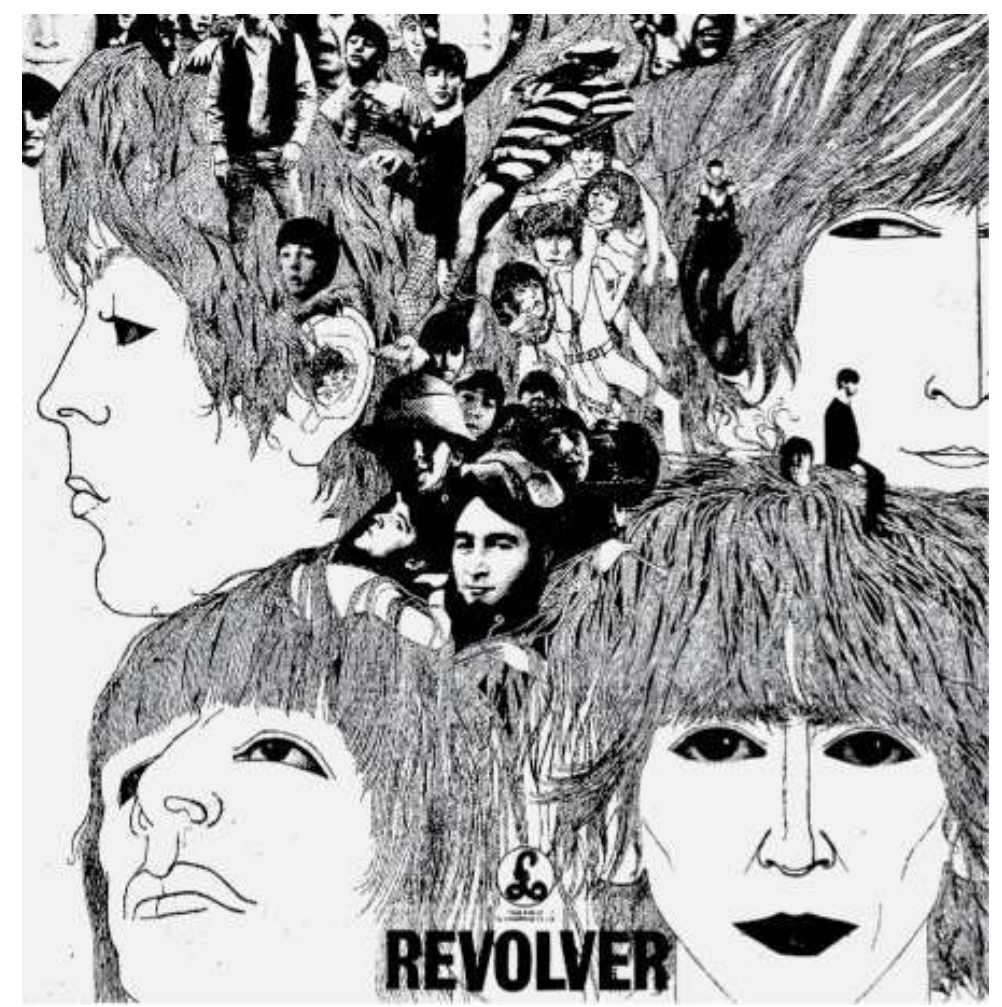

Quel est donc la bonne clé de lecture du morceau? Le problème vient de ce que Love You To naît de la rencontre de deux cultures musicales et, par conséquent, elle ne peut, de par sa nature même, être attribuée exclusivement à l'une ou à l'autre. En plus l'une des deux, l'occidentale, celle qui réalise le métissage, est prédominante (Harrison lui-même situe la chanson dans ce contexte): c'est seulement dans cette optique de renvois complexes qu'il est possible de faire une analyse correcte de la production née du crossover.

En fait, une condition pour que des éléments «autres» puissent être insérés dans un contexte pop est la «possibilité d'accueillir» - au moins partiellement - des sons, des formes et des tournures comme «familiers». En même temps, on assiste au contraire à l'appropriation de structures et d'instruments hindoustanis justement parce qu'ils sont considérés comme «nouveaux» et souvent exotiques. Comme nous l'avons déjà indiqué, ce processus se situe dans un contexte de recherche de sonorités différentes et d'expérimentation avec la forme-chanson.

Rien qu'à considérer son organisation, on voit déjà combien Love You To se situe dans une zone frontière entre la culture pop occidentale et celle de la musique indienne. Les Beatles y utilisent en effet une guitare électrique ou le tambourin sur l'off beat (typiques de l'idiome rock) et, en même temps, un sitar ${ }^{22}$, un tanpura et les tabla.

54 L'aspect du timbre reste cependant un élément dont toute analyse correcte du phénomène se doit de tenir compte. L'expérimentation des sonorités du sitar, qui se situent à mi-chemin entre l'électrique et l'acoustique grâce à l'effet «métallique» créé par la résonance des cordes sympathiques, a probablement été la raison principale qui a mené 
au crossover entre la musique pop occidentale et la tradition hindoustanie et qui a conduit les Beatles à adopter cet instrument avant même les enregistrements de Love You To. On peut en dire autant du bourdon du tanpura qui, à l'oreille du musicien pop, se présente comme le substratum acoustique idéal pour créer une dimension "suspendue», psychédélique. Dans Love You To on a vu à quel point ces deux instruments sont utilisés consciemment par Harrison pour correspondre aux préceptes de la tradition indienne. Toutefois cette réflexion doit être valable pour les autres membres du groupe, qui, étant en fait étrangers à la culture et à la théorie musicale hindoustanies, doivent avoir perçu la chanson en terme d'expérimentation plutôt sonore que formelle ${ }^{23}$.

En ce qui concerne la mélodie du morceau, nous avons déjà brièvement indiqué combien l'utilisation du kafi tata pouvait s'expliquer par son analogie avec la gamme blues, elle aussi dotée d'un troisième et d'un septième degrés abaissés, et très utilisée dans le pop/ rock. D'autre part, on ne peut pas non plus sous-estimer le processus inverse, à savoir que telle échelle modale a pu être employée à cause de la fascination pour certaines sonorités, pour certaines lignes mélodiques à saveur «exotique».

Sur le plan rythmique également, la possibilité de percevoir une structure orientale comme semblable à un mètre occidental a entraîné l'utilisation du tintal. Il a été interprété comme un $4 / 4$ ou comme un 424/4 dans lequel chaque quart correspond à une pulsation (matra) du tala exécuté. Ce qui donne une distribution des accents différente de la pratique originale hindoustanie: en effet, en accentuant le premier quart de chaque battue en $4 / 4$, la douzième pulsation du tintal perd sa fonction du khali (accent non existant). Cependant, du moment où le tabliste respecte la structure du theka dans Love You To, on assiste à la création d'une véritable «superposition» de conceptions rythmiques.

Ce fait illustre clairement et confirme qu'à la base du métissage effectué par les Beatles entre la musique pop et la musique de l'Inde du Nord, il existe une forte ambiguïté entre les éléments des deux cultures.

C'est pour cette raison que, par exemple, même une structure comme la forme responsoriale, largement répandue aussi bien en Inde (le sawal-jawab) que dans la musique pop (le call \& response du blues) et présente dans Love You To dans les dialogues entre voix et sitar, figure parmi les éléments ayant servi de pont entre l'Est et l'Ouest, justement à cause de leur capacité naturelle d'accueil.

59 Pour conclure, dans toute recherche sur les mécanismes du métissage entre la musique pop et la musique hindoustanie, l'analyse des éléments formels, mélodiques et rythmiques du morceau étudié reste fondamentale car elle permet d'identifier des structures, des formules, des gammes ou des cycles rythmiques venus de la musique hindoustanie et de comprendre de quelle manière ils ont été insérés dans le contexte de la chanson pop occidentale. D'autre part on ne doit pas négliger, quand cela est possible, la reconstruction de l'histoire du morceau dans le studio d'enregistrement, en se référant aux comptes rendus et aux dires des protagonistes eux-mêmes, pour pouvoir évaluer le niveau de conscience avec lequel furent utilisés les éléments «autres». La reconstitution de la genèse de la chanson pop, souvent très liée à l'anecdote, constitue une perspective d'étude négligée jusqu'à maintenant, mais qui se révèle être une clé pour comprendre comment des musiciens rock ont accueilli les éléments indiens et comment ils se sont rapportés à eux à travers la musique pop occidentale. Une recherche de ce type s'est avérée indispensable dans le cas de Love You To pour connaître, par exemple, la position 
de Harrison face à sa chanson et pour pouvoir «orienter» de façon appropriée le cadre dans lequel se situe le morceau et le débat auquel il a donné lieu.

Quant aux Beatles et à leur apport, il est évident qu'ils constituent un cas particulier par la qualité de leur intervention, grâce surtout à l'entremise consciente de George Harrison. Ils ont en outre ouvert la voie de la contamination à toute la musique pop occidentale qui suivit. Dans leur sillage, les productions où apparaissent des instruments et des sonorités hindoustanis se sont multipliées et même après le premier grand impact de la "great sitar explosion» leur écho a continué d'influencer le paysage musical. C'est seulement dans ce double rôle d'expérimentateurs et de promoteurs qu'il est possible de comprendre pleinement quels furent leur apport et leur importance dans le métissage entre la musique pop et la world music.

\section{BIBLIOGRAPHIE}

BAUMANN Max Peter, ed., 1992, World Music - Musics of the Worlds. Aspects of Documentation, Mass Media and Acculturation. International Institute for Traditional Music; Berlin: Wilhelmshaven: Florian Noetzel Verlag.

DAVIES Hunter, 1985, The Beatles. The only authorized biography, updated and with additional illustrations. London: Jonathan Cape.

FARRELL Gerry, 1988, «Reflecting surfaces: the use of elements from Indian music in Popular Music and Jazz», Popular Music 7/2: 189-204.

FARRELL Gerry, 1997, Indian Music and the West. Oxford: Clarendon Press.

GOTTLIEB Robert S., 1977, The major traditions of North Indian tabla drumming. Salzburg: Musikverlag Emil Katzbichel.

HARRISON George, 1982, I me mine. London: W.H.Allen \& Co. Ltd.

HERTSGAARD Mark, 1995, A day in the life. La musica e l'arte dei Beatles. Milano: Baldini \& Castoldi s.r.l.

JUNIUS Manfred M., 1974, The Sitar. The instrument and its Technique, Wilhelmshaven: Heinrichshofen's Verlag.

KAUFMANN Walter, 1993, The Ragas of North India. Sittingbourne: Asia Publishing House Ltd.

KEIL Charles FELD Steven, 1994, Music Grooves: essays and dialogues. Chicago and London: The University of Chicago Press.

KIPPEN James, 1988, The tabla of Lucknow: a cultural analysis of a musical tradition, Cambridge: Cambridge University Press.

LEANTE Laura, 1998-99, I Beatles e la musica indostana. Analisi di un'appropriazione. Tesi di laurea inedita, Università degli Studi di Roma «La Sapienza».

LEWISOHN Mark, 1988, The Beatles' Complete recording sessions. London: The Hamlyn Publishing Group Limited. 
MAC DONALD Ian, 1994, Revolution in the head: the Beatles' records and the Sixties. London: Fourth Estate.

MARTIN Denis-Constant, 1996, «Who's afraid of the big bad world music? (Qui a peur des grandes méchantes musiques du monde?)», Cahiers de musiques traditionelles 9: 3-21.

MELLERS Wilfrid, 1973, Twilight of the gods: the Beatles in retrospect. London: Faber \& Faber.

O'GRADY Terence, 1983, The Beatles: a musical evolution. Boston: Twayne Publishers.

RECK David, 1985, «Beatles Orientalis», Asian Music (Journal of the Society for Asian Music) XVI: 83-149.

RILEY Tim, 1988, Tell me why. A Beatles commentary. Oxford: The Bodley Head Ltd.

RUSSELL Jeff, 1982, The Beatles album file and complete discography. London: Blandford Press.

SHANKAR, Ravi, 1969, My music, my life. New Delhi: Vikas Publishing House Pvt Ltd.

SORRELL Neil, 1980, Indian music in performance, Manchester: Manchester University Press.

WADE Bonnie, 1979, Music in India: the classical traditions, Englewood Cliffs, N.J.: Prenctice-Hall.

\section{NOTES}

1. Denis Constant Martin (1996) s'est intéressé au problème de la définition du terme «world music», proposant une perspective historico-sociologique et économique du phénomène. Il montre que l'ethnomusicologie est la discipline idéale pour une étude des sens de la «world music » dans les sociétés occidentales car «il semble que, sans négliger leurs champs d'études propres, les ethnomusicologues puissent apporter des connaissances et porter des jugements, indispensables les unes et les autres à une meilleure compréhension de ce que signifie la popularité des «musiques du monde» (Ibid.: 21).

2. Une référence importante à ce sujet est le Music Grooves de Charles Keil et Steven Feld (1994) qui pose clairement le thème du rapprochement de l'ethnomusicologie avec la problématique liée à la pop et la world music. Sur la base de leur intérêt plus strictement musicologique, les auteurs proposent en fait aussi une réflexion de type sociologique sur le jazz, la pop, la world music et sur l'impact qu'ont eu, et ont toujours, sur elles les nouvelles technologies, les médias et la réalité du marché du monde occidental. Sur les mêmes thèmes, voir aussi Max Peter Baumann ed. (1992).

3. Shankar 1982: 92.

4. Norwegian Wood fut publiée dans l'album Rubber Soul en 1965.

5. Le critère que j'adopte pour une identification non susceptible d'erreur des chansons des Beatles où l'influence indienne est certaine, est de considérer les morceaux «physiquement» imprégnés par les instruments hindoustanis. La liste des pièces qui en résulte est la suivante: Norwegian Wood (1965); Tomorrow Never Knows, Love You To, Strawberry Fields Forever (1966); Lucy in The Sky With Diamonds, Getting Better, Within You Without You (1967); The inner light, Across the Universe (1968).

6. Dans la plupart des cas, la bibliographie sur les Beatles est constituée de textes qui envisagent, souvent sous forme de compilation, leur production en général, fournissant tout au plus quelques notions sur les instruments indiens utilisés (par exemple, Riley 1988; Mac Donald 1994; Hertsgaard 1995). Dans ces textes l'absence d'une perspective ethnomusicologique a parfois entraîné la diffusion d'erreurs grossières sur la nature même des instruments (Russell 1982: 69,72,82-83, 92) ou l'omission du sujet (Mac Donald 1994: 152). 
7. L'analyse rythmique de Love You To proposée par Reck (1982: 102) est forcée: il étend en effet son identification d'influences indiennes à des aspects qui sont en réalité le fruit d'une adaptation de cycles rythmiques hindoustanis aux exigences du morceau pop.

8. Cf. Leante 1998-1999.

9. Il n'existe pas d'édition officielle des transcriptions des chansons des Beatles. Les transcriptions publiées présentent souvent les limites d'une simplification excessive (par exemple: The Beatles Complete. Piano/Organ/Vocal Edition, London: Wise Publications. 1983). Par ailleurs, même les partitions plus détaillées (par exemple: The Beatles Complete Scores, London: Wise Publications. 1993) reprennent souvent des erreurs au sujet de l'identification correcte des éléments indiens et souffrent surtout d'une réduction excessive due à l'usage de l'écriture occidentale.

10. Lewisohn 1988: 72; Pour les tabla voir: Gottlieb 1977; Kippen 1988; Sorrell 1980: 40-43; Wade 1991: 135-139.

11. "...There is the use of harmonics in the opening slow passage. Harmonics are never used as a musical device on the sitar (they are used for tuning purposes).» (NdT)

12. Voir Lewisohn 1988: 72.

13.

Qui se ramène au theka du tintal:dhadhindhindhadhadhindhindhadhatintintatadhindhindha 1 $2345678910111213141516 \mathrm{sam}$

14. Lewisohn 1988: 73.

15. Pour une définition du raga, voir: Sorrell 1980: 93-103; Farrell 1988: 191.

16. Pour une définition du tata voir Sorrell 1980: 94-96.

17. La gamme ci-dessous sert seulement à illustrer le profil des intervalles du kafi tata et n'indique pas la hauteur absolue des sons.

18. «[...] there are many aspects of this song which are well within the tradition of Western misunderstanding of Indian music, even if the setting was unprecedented for a popular song. [...] the greatest misunderstanding comes in the vocal delivery, which reflects the notion that to sound Indian, one must sing flat and with a nasal tone, rather than fluently and expressively. In terms of structure, Harrison's song undoubtely gives a simple sketch of the larger-scale structure of an Indian performance which are rendered meaningless without the detail." (Farrell, 1997: 184). (NdT)

19. Farrell 1988: 194.

20. «The complete essence of the classical Indian tradition» (O'Grady 1983: 101).

21. «Real Indian classical songs are so much different from the sort of Indian influenced pop songs which I have been turned out over here. They're just ordinary pop songs, with a little bit of Indian background.I'm not sure about the ones I've written. Looked at from another person's point of view, then as pop songs I like them [...]" (Harrison, cit. in Davies, 1985: 409). (NdT)

22. Le sitar est souvent investi d'un rôle nouveau par les musiciens pop à cause de son aspect qui le fait apparaître comme une "guitare exotique».

23. A ce propos, il est intéressant de rappeler ce que dit Farrell au sujet de la voix de Harrison dans Love You To: il explique combien justement le timbre nasal appartient aux clichés à travers lesquels les occidentaux se représentent la musique indienne. (Farrell 1997: 184). 


\section{RÉSUMÉS}

Le métissage entre la musique indienne et la musique pop, très présent aujourd'hui dans la production discographique occidentale, trouve ses racines dans les années soixante qui, dans un contexte plus vaste de fascination pour l'exotique, virent naître le désir d'expérimenter de nouvelles sonorités. Au cœur de ce phénomène, les Beatles se distinguent non seulement parce qu'ils furent parmi les principaux promoteurs de cette tendance, mais aussi parce qu'ils ne se cantonnèrent pas à l'utilisation d'instruments hindoustanis pour leur seule sonorité: leur expérimentation en effet tenait également compte de l'aspect formel, mélodique et rythmique. Love You To se présente comme la somme de tous ces aspects et constitue un sujet d'analyse idéal pour comprendre de quelle façon s'est effectué le crossover à la base duquel on trouve une forte ambiguïté entre ce qui est adopté intentionnellement par le musicien pop et ce qui, perçu comme «familier», est absorbé inconsciemment.

Quels sont donc les paramètres à utiliser pour une bonne lecture du morceau? L'étude de Love You To se situe dans une «zone frontière» entre la musique pop et l'ethnomusicologie et nécessite donc les instruments de ces deux domaines. Si l'on ne peut négliger le recours à l'analyse musicale pour comprendre comment, du point de vue technique, s'est effectuée la mutation, il faut aussi mener l'enquête sur les sources et reconstituer la genèse de la chanson et le processus de son enregistrement afin de mieux évaluer le degré de conscience du musicien dans le métissage qu'il était en train de réaliser.

\section{AUTEURS}

\section{LAURA LEANTE}

Laura Leante est doctorante en «histoire et analyse des cultures musicales» à l'Université La Sapienza de Rome. Sa recherche est centrée sur les influences exercées par la musique indienne sur la musique populaire occidentale, en particulier au sein de la production des Beatles. 